\title{
Problems with Measuring Beam Size of a Focused Ion Beam System by Means of the Rise-Distance
}

\author{
J. Orloff* and L. Y. Roussel** \\ * FEI Company, 5350 NE Dawson Creek Drive, Hillsboro, OR 97124, USA \\ ** FEI Company, Building AAE, PO Box 80066, 5600 KA Eindhoven, The Netherlands
}

The rise-distance method is frequently used to estimate the beam size in a focused ion beam (FIB) system. At small currents $\Leftrightarrow$ small beam defining aperture most of the current density is described by a Gaussian shape and the beam rise-distance can be related to the full width at half maximum (FWHM), which gives an intuitive meaning to beam size. Thus the method is a reasonable way to define system performance. However, there are numerous pitfalls in this method which we want to emphasize.

The rise-distance of a beam is measured by sweeping the beam across a real or simulated knife edge which is defined as a region on a specimen where the secondary electron yield changes rapidly in a distance small compared to the beam size. The change in secondary electron current $\left(\mathrm{I}_{\mathrm{sec}}\right)$ is measured as a function of distance and used to define a beam size (see Figure 1). For example, the distance over which the secondary electron current changes from $20 \%$ to $80 \%$ of its full value is often used as a measure of beam size.

The main problems associated with this method are: 1) the knife edge erodes under ion bombardement - this will be of particular importance in the case of a heavy ion gallium FIB and affects the imaging resolution seriously $[1,2]$; 2) the secondary electron yield changes as the specimen is eroded and may not even be constant irrespective of ion bombardement and implantation; 3) most significantly, at low currents there will be severe fluctuations in $I_{\sec }$ due to noise in the ion beam, resulting in a poor statistics. This last issue is most troublesome and requires that many passes of the beam be made in order to achieve good statistics, which is in conflict with the requirement that the specimen erosion issue be minimized by using as few coulombs as possible to make the measurement. As a result, it is necessary to produce an image of the specimen and analyze the rise distances of many (thousands) of individual passes across various parts of the knife edge. The result will be a range of rise-distances which we have found can vary by over a factor of 3. An example is shown in Figures 2 and 3 where, in a measurement with a $1 \mathrm{pA} \mathrm{Ga}{ }^{+}$beam at 30 $\mathrm{keV}$, the best fit to a single pass is $1.8 \mathrm{~nm}$ while the average over thousands of passes is $4 \mathrm{~nm}$. The statistically averaged edge measurement, performed with appropriate thresholds, is a realistic estimate of the beam size while the single edge number is at risk of providing an unrealistic result. For this reason, it is necessary to determine whether a beam size estimate is an average or the result of choosing the best data from among a set of many.

References

[1] J. Orloff, L.W. Swanson and M. Utlaut, "Fundamental Limits on Imaging Resolution in Focused ion Beam Systems", J. Vac. Sci. Tech. B14 (1996) p 3759

[2] V. Castaldo, C.W. Hagen, B. Rieger and P. Kruit, "Sputtering limits versus signal-to-noise limits in the observation of Sn balls in a $\mathrm{Ga}^{+}$microscope," J. Vac. Sci. Tech. B26 (2008) p 2107 


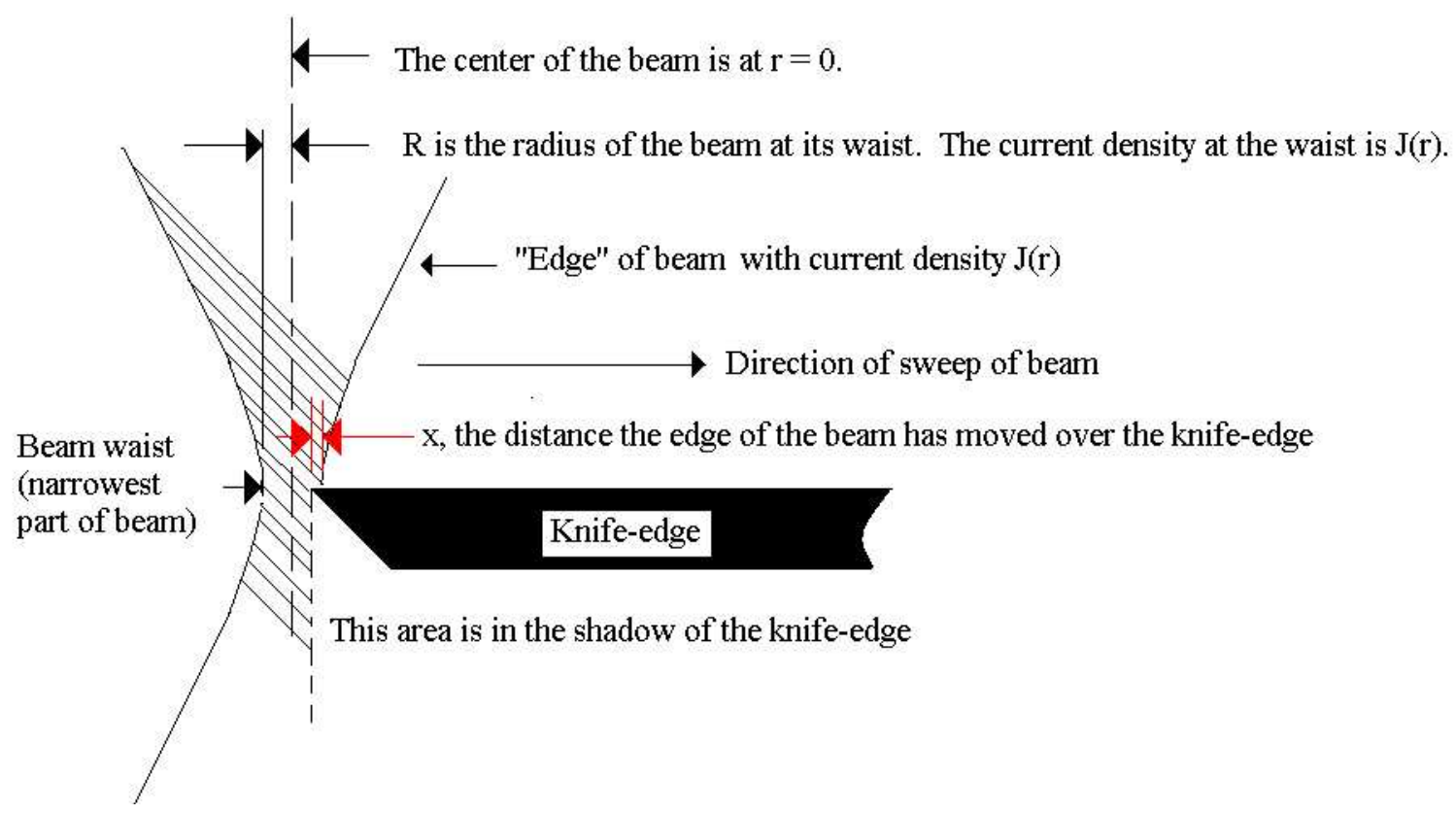

FIG. 1. The concept of measuring beam size by the rise-distance method is shown schematically. As the beam passes across the knife edge the secondary electron current rises from 0 to its full value $I_{\text {sec. }}$. Beam size can be defined as the distance $x$ over which $I_{\text {sec }}$ rises from $20 \%$ to $80 \%$ of its full value, for example.

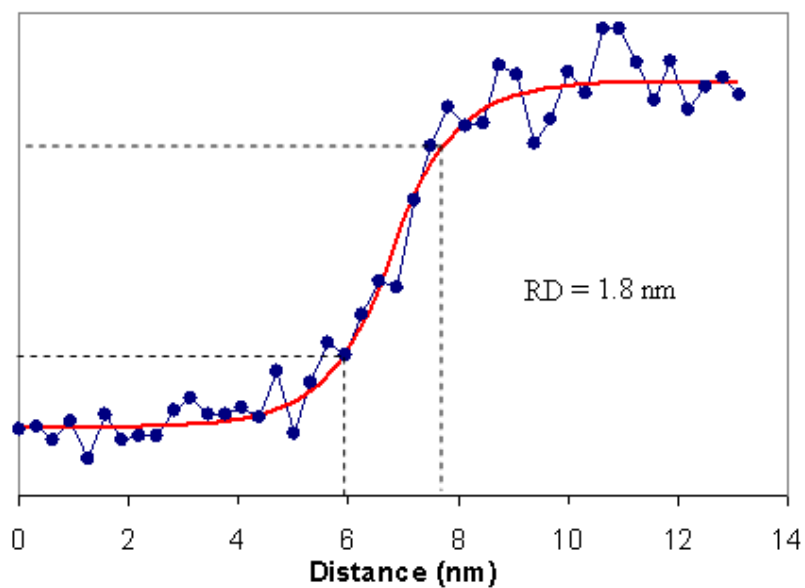

FIG. 2. The rise-distance of a $1 \mathrm{pA} \mathrm{Ga}^{+}$ion beam at $30 \mathrm{keV}$ over a knife edge. The dots show the statistical variation in a single pass of the beam over the knife edge. The smooth curve is the best fit to the data, showing a $20 \%$ - $80 \%$ rise-distance of $1.8 \mathrm{~nm}$. (Courtesy M. Maazouz, FEI Company).

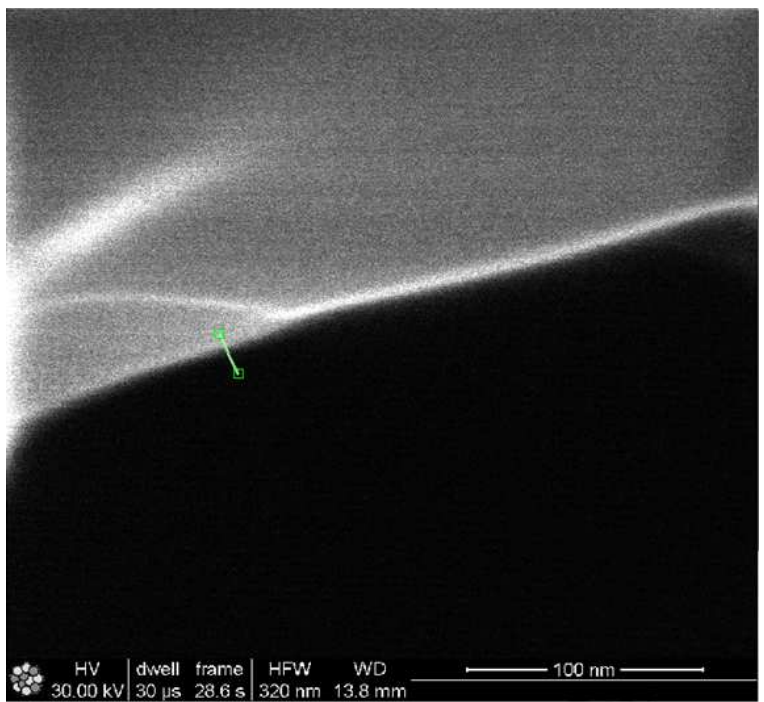

FIG. 3. Knife edge on a graphite sample, imaged by a $30 \mathrm{kV}, 1 \mathrm{pA}$ focused $\mathrm{Ga}^{+}$beam (courtesy M. Maazouz, FEI Company). The line shows where a single measurement was made. 